\title{
Aesthetic subunits of the trunk: Anatomy and behavior of skin and adipose tissue in each unit for better outcomes in liposuction
}

\author{
Sanjay Parashar, Aniketh Venkataram ${ }^{1}$ \\ Cocoona Center for Aesthetic Transformation and Day Surgery Hospital, Dubai, UAE, 'Department of Plastic and Aesthetic \\ Surgery, Venkat Center for Skin and Plastic Surgery, Bengaluru, Karnataka, India
}

Address for correspondence: Dr. Sanjay Parashar, Cocoona Center for Aesthetic Transformation, Dubai, UAE.

E-mail: sanjay@cocoona.ae

\begin{abstract}
Background: Liposuction and abdominal contouring is one of the most common procedures performed by plastic surgeons worldwide. Surprisingly, there has been few attempts at dividing the abdomen into subunits to delineate and aid in this endeavour. We have evolved a system of abdominal aesthetic subunits which has enabled us to achieve high quality results. Materials and Methods: A system of 8 subunits of the abdomen was evolved after analysing 1000 cases: Upper midline, upper rectus, lower rectus, pubic, Lateral abdomen, Lumbar Posterior midline and Bra Roll. Results: The system was used in 2000 cases with good results. The system enabled the performance of tailored liposuction in each area, with greater accuracy, better results and fewer complications. Conclusion: Our success with this classification validates its use, and makes liposuction more scientific and effective.
\end{abstract}

\section{KEY WORDS}

Abdomen; aesthetic subunits; liposculpturing; liposuction; ultrasonic assisted

\section{INTRODUCTION}

iposuction and abdominal contouring are one of the most common procedures performed by plastic surgeons worldwide. Earlier techniques involved the removal of fat from the abdomen circumferentially without much variation in different regions..$^{[1]}$ However, the advancement of technology and expertise has led to the dawn of high definition liposuction and body sculpting. ${ }^{[2]}$ Surprisingly, there have been few attempts at dividing the

\begin{tabular}{|l|l|}
\hline \multicolumn{2}{|c|}{ Access this article online } \\
\hline Quick Response Code: & Website: \\
\hline
\end{tabular}

abdomen and trunk into subunits to delineate and aid in this endeavour. The only significant classification existing thus far is that by Matarasso and Wallach. ${ }^{[3]}$ We have evolved a system of abdominal aesthetic subunits based on the anatomy of skin and fat, as well as requirements of liposuction in each different area. This has enabled us to achieve high-quality results, while at the same time improving education and communication.

This is an open access journal, and articles are distributed under the terms of the Creative Commons Attribution-NonCommercial-ShareAlike 4.0 License, which allows others to remix, tweak, and build upon the work non-commercially, as long as appropriate credit is given and the new creations are licensed under the identical terms.

For reprints contact: reprints@medknow.com

How to cite this article: Parashar S, Venkataram A. Aesthetic subunits of the trunk: Anatomy and behavior of skin and adipose tissue in each unit for better outcomes in liposuction. Indian J Plast Surg 2018;51:115-22. 


\section{MATERIALS AND METHODS}

Records were collected, and 1000 cases of truncal liposuction were analysed. Abdominoplasty procedures were excluded. The following classification of aesthetic subunits of the trunk was devised [Figures 1 and 2]

1. Upper midline-overlying the linea alba from the xiphisternum to the umbilicus

2. Upper rectus-overlying the rectus muscles from lower costal margin to umbilicus on each side

3. Lower rectus-single unit over both the recti from the umbilicus to the upper pubic hairline

4. Lateral abdominal-overlying the oblique muscles, on either side of the recti from the costal margin to the inguinal ligament, until the midlateral line. This was further divided into two parts:

4a.Upper part of lateral abdomen overlying costal region

4b.Lower part of lateral abdomen overlying the oblique muscles also part of flanks

5. Pubic-below the pubic hairline

6. Lumbar-overly the lumbar muscles from the costal margin to the gluteal region, until the paraspinous muscles posteriorly

7. Posterior midline-overlies the sacrum, spinous processes and the paraspinal muscles

8. Bra roll-above the lumbar unit, extending into the infraaxillary region.

This classification allows us to identify each aesthetic zone of the trunk. The pattern of fat distribution and overlying skin quality varies in different zones. The underlying musculoskeletal structure gives an aesthetically pleasing shape to the torso. This shape gets altered with fat accumulation. While some patient with excess fat in all the zones may still have aesthetically curved torso, others may lose the contour due to irregular and uneven fat distribution pattern. Each zone has a characteristic shape based on the underlying structure and overlying skin tone. Often, there is more volume of fat deposited in the lower abdomen and lumbar region than upper abdominal zones. The fat texture in upper zones is more fibrous and compact, hence has more risk of irregularities following liposuction. The skin in the lower abdomen is loosely anchored and skin sagging is more common in the lower abdomen.

Following this, a total of 2000 procedures were performed using the system from 2010 to 2018. All patients were evaluated with this classification. The subunits were marked on patients preoperatively [Figure 3]. All cases were done under tumescent anaesthesia with intravenous sedation. Infiltration was performed uniformly and symmetrically in all the zones. Zone 1 and Zone 2 have tough fibrofatty tissue architecture; hence, it is more painful and tough to inject. The risk of bleeding is more in upper abdominal zones. The lower abdomen has a

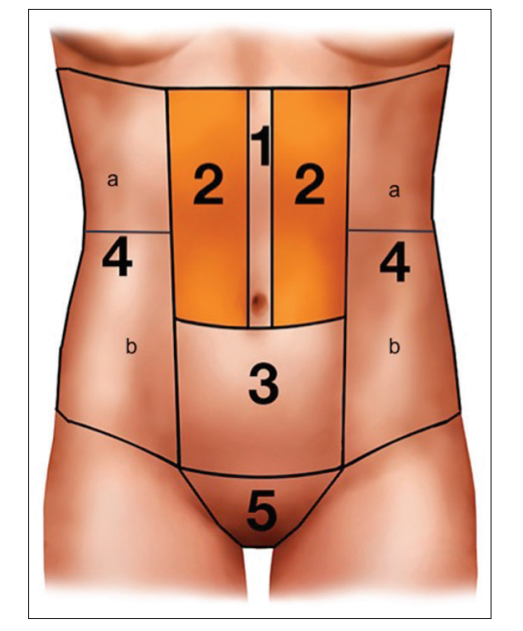

Figure 1: Aesthetic Subunits of Anterior abdomen

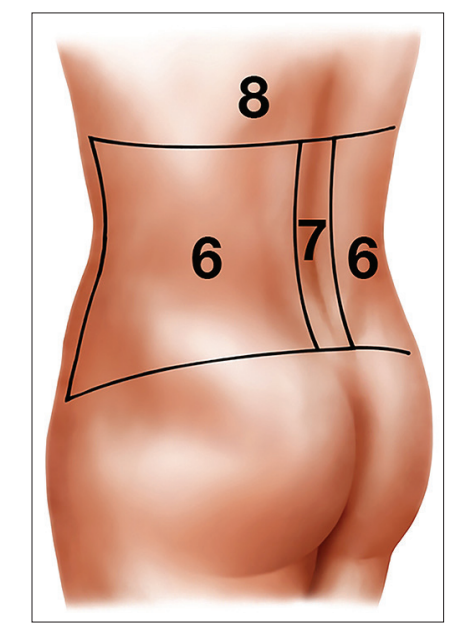

Figure 2: Aesthetic subunits of posterior trunk

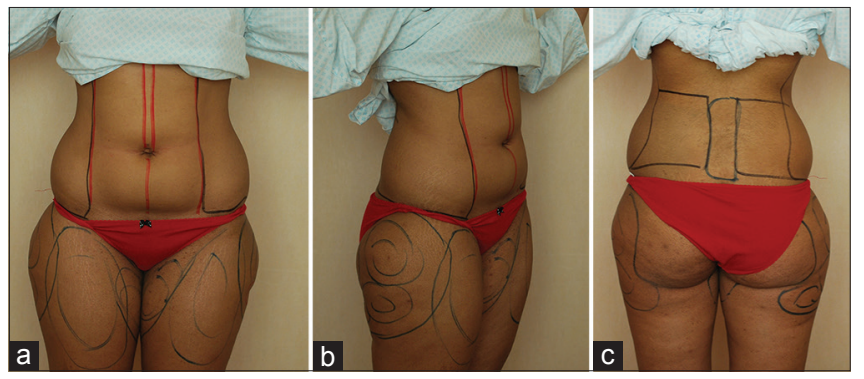

Figure 3: (a) Marking of Aesthetic subunits of Anterior Trunk in a female for Body sculpting. (b) Marking of Aesthetic subunits of Lateral Trunk in a female for body sculpting. (c) Marking of Aesthetic subunits of Posterior Trunk in a female for body sculpting 
loose subscarpal fatty layer that allows easy infiltration and hence is less painful. The posterior part of the trunk, particularly Zones 6 and 7 also have tough fibrofatty tissue with dense deep adhesions. It requires thorough infiltration for adequate anaesthesia and bloodless aspiration. A thorough and effective infiltration takes about 25-30 min.

Following infiltration ultrasonic emulsification is performed. Upper abdomen requires more ultrasonic energy and time to emulsify than lower abdomen. A proper undermining using ultrasonic device allows uniform skin retraction. Thorough emulsification helps minimise bleeding, bruising, pain and allows good skin retraction. The average recommended time is $1 \mathrm{~min} / 100 \mathrm{ml}$ of infiltrate [Figure 4].

Then, liposuction was performed using $3 \mathrm{~mm}, 4 \mathrm{~mm}$ Mercedes type cannula. Zone 1 needs thorough liposuction to achieve a nice midline groove. Zone 2 has tough, fibrous fat and needs careful liposuction as there is a higher risk of accidental deeper injury and a higher risk of lumpiness or skin irregularities. The cross tunnelling method also helps prevent linear irregularities. The skin over this zone has tight adhesions to underlying costal region and hence requires an adequate release for good retraction. Zone 3 is easier to aspirate, but through deep and superficial liposuction is required to achieve a good skin retraction. Zone $4 \mathrm{a}$ has tough, fibrous adhesion overlying the costal region that requires proper undermining and release. Adequate deep fat extraction in this zone gives a nice silhouette to the lateral part of the abdomen. Zone $4 \mathrm{~b}$ has soft fat overlying oblique muscles and has two distinct layers above and below the Scarpa's fascia.

A simultaneous liposuction for Zone 5 over the pubic zone is also important as the patient may have a fat bulge if they gain weight postoperatively.

Zone 6 has two distinct layers of fat deep and superficial, deep fat is embedded in the lumbar triangle and if not adequately removed the bulge may persist or recur with weight gain. To achieve a highly desired lordotic lower back shape in women, both these compartments must be treated.

Zone 7 has the 'triangle of Venus' inferiorly and midline depression overlying the spinous process. It is ideal to completely remove fat in the Venus triangle and create a midline groove if it is ill-defined. Zone 8, i.e., the bra roll area also needs addressing as it is a common site of recurrence and exaggeration if patient continues to gain weight.

During the procedure, the amount of infiltrate used, as well as the amount aspirated was noted for each of the subunits [Table 1]. Zones also allow symmetrical and uniform aspiration. All the incision ports were left open to drain. A compression dressing is applied for the first $24 \mathrm{~h}$ followed by pressure garment. The first stage pressure garment was adviced for 4-6 weeks depending on skin elasticity. A second stage pressure garment called 'spanx' was recommended for 2-3 months. The foam was applied to different areas for additional compression. Postoperatively, any asymmetries or complications were also documented using these subunits. The follow-up protocol included schedules on day 2, day 5, 1 week, 3 weeks, 2 months, 4 months, 6 months, 1 year and 2 years. Every visit measurements was taken and photography done.

\section{RESULTS}

Patients who were treated using this method of aesthetic subunits were satisfied with their results. The results were

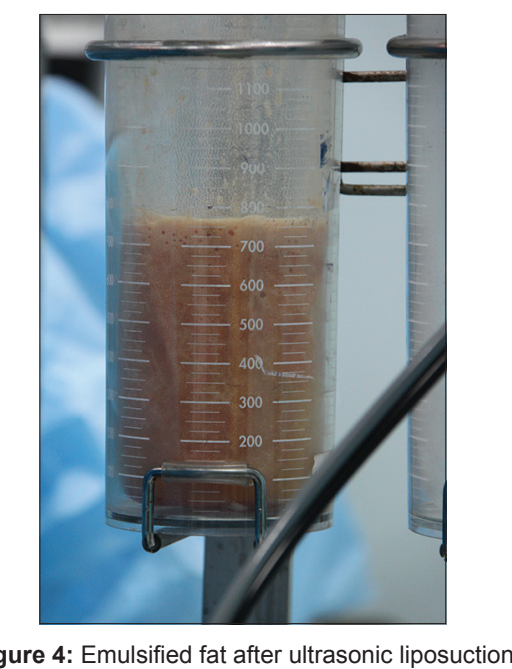

Table 1: Intraoperative assessment of liposuction using subunits

\begin{tabular}{lcccc}
\hline Subunit & $\begin{array}{c}\text { Infiltration } \\
(\boldsymbol{m})\end{array}$ & $\begin{array}{c}\text { VASER } \\
\text { energy }\end{array}$ & $\begin{array}{c}\text { VASER } \\
\text { duration }(\boldsymbol{m i n})\end{array}$ & $\begin{array}{c}\text { Aspirate } \\
(\boldsymbol{m})\end{array}$ \\
\hline I, II & 500 & $80 \%$ & 4 & 400 \\
III & 800 & $100 \%$ & 8 & 700 \\
IV (each side) & 400 & $80 \%$ & 3 & 250 \\
V & 100 & $80 \%$ & 1 & 50 \\
VI (each side) & 400 & $80 \%$ & 4 & 300 \\
VII & 100 & $80 \%$ & 1 & 50 \\
VIII (each side) & 200 & $100 \%$ & 2 & 150 \\
Total & $3500 \mathrm{ml}$ & & 32 & 2450 \\
\hline
\end{tabular}

Indian Journal of Plastic Surgery Volume 51 Issue 2 May-August 2018 
assessed by physical examination, serial photographs, measurements and patient satisfaction [Figures 5-7]. The use of these subunits enabled us to perform different degrees of liposuction with different aims in each of the subunits which improved overall aesthetics. This can be applied in all weight category patients who are fit for the liposuction procedure. In more athletic individuals with good skin quality results are far superior. In moderately overweight patients, good curvature can be achieved three-dimensionally. Use of the subunits also had the following advantages:

- Ease in planning

- Ease in calculating infiltration required by subunit

- Ease in calculating volume extracted from each subunit

- Avoidance of errors-particularly asymmetry

- Ease in follow-up.

Common complications in our series were skin unevenness in the upper abdomen, mild asymmetry in the lower

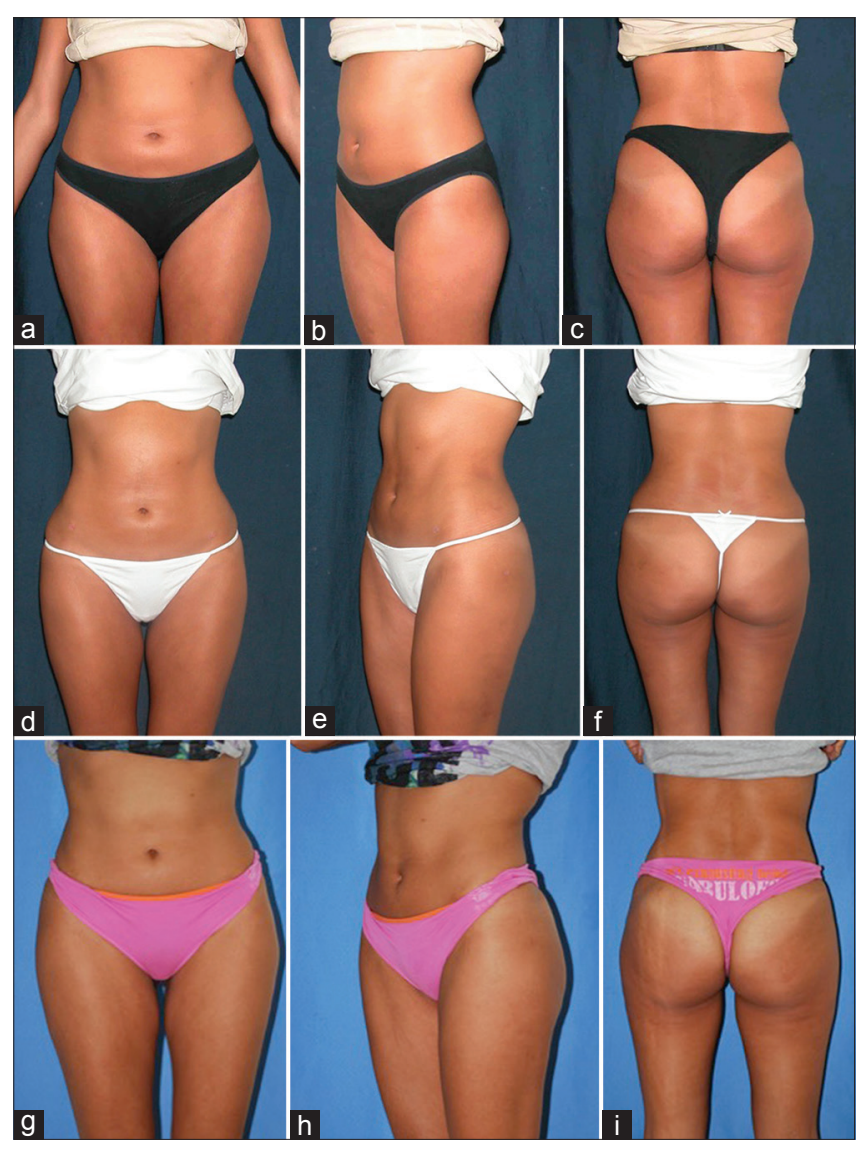

Figure 5: (a)Anterior preoperative views of young female with body mass index 24. (b) Oblique Preoperative views of Young female with body mass index 24. (c) Posterior Preoperative views of Young female with body mass index 24. (d) 6 months Anterior Postoperative views of same patient. (e) 6 months oblique Postoperative views of same patient. (f) 6 months Posterior Postoperative views of same patient. (g) 3 years anterior postoperative of the same patient. (h) 3 years oblique Postoperative of the same patient. (i) 3 years posterior postoperative of the same patient abdomen, visible scars and hyperpigmentation. There were no incidences of seroma, skin loss, infection, etc.

\section{DISCUSSION}

The use of aesthetic subunits is well known in plastic surgery. Gonzales-Olloa designed subunits of the face. ${ }^{[4]}$ Scott Spear described subunits of the breast ${ }^{[5]}$ and Mendieta described gluteal subunits. ${ }^{[6]}$

There have previously been a few attempts at devising an aesthetic classification of the abdomen. The best known of these is that by Matarasso et al., which described seven subunits in females: upper abdomen, Lower abdomen, umbilicus, flanks, mons, sacral area and bra roll. Moreover, 6 subunits in men: upper abdomen, lower abdomen, umbilicus, flanks, mons sacral area. ${ }^{[3]}$

It was Mentz and Ersek who pointed out that traditional lipoplasty techniques fail to achieve the desired results of abdominal contouring. ${ }^{[7,8]}$ The Mentz technique of abdominal etching used differential lipoplasty to detail abdominal musculature. However, it was designed for male bodybuilders and only the anterior abdominal wall. In 2003, Hoyos presented a new technique of high definition liposculpture. ${ }^{[2]}$ This elevated the concept of etching to a three dimensional approach. It also integrated the differing requirements of the male and female body. This became the gold standard for abdominal contouring, and with its advent we found the Matarasso classification lacking. Our system of aesthetic subunits of the trunk

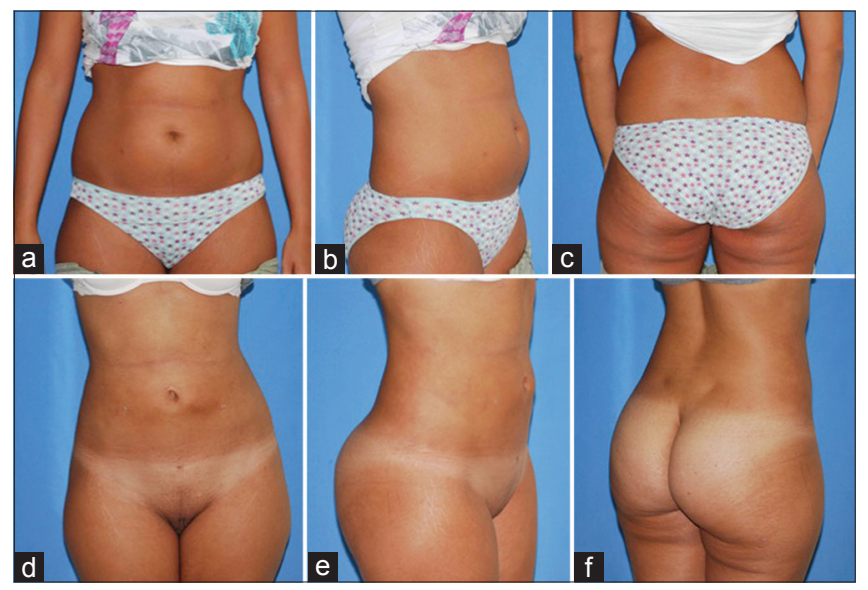

Figure 6: (a) Anterior preoperative views of young female patient with body mass index 26 for body contouring, (b) Oblique preoperative views of young female patient with body mass index 26 for body contouring, (c) Posterior preoperative views of young female patient with body mass index 26 for body contouring, (d) Anterior Postoperative views after 1 year, (e) Oblique Postoperative views after 1 year, (f) Posterior Postoperative views after 1 year 


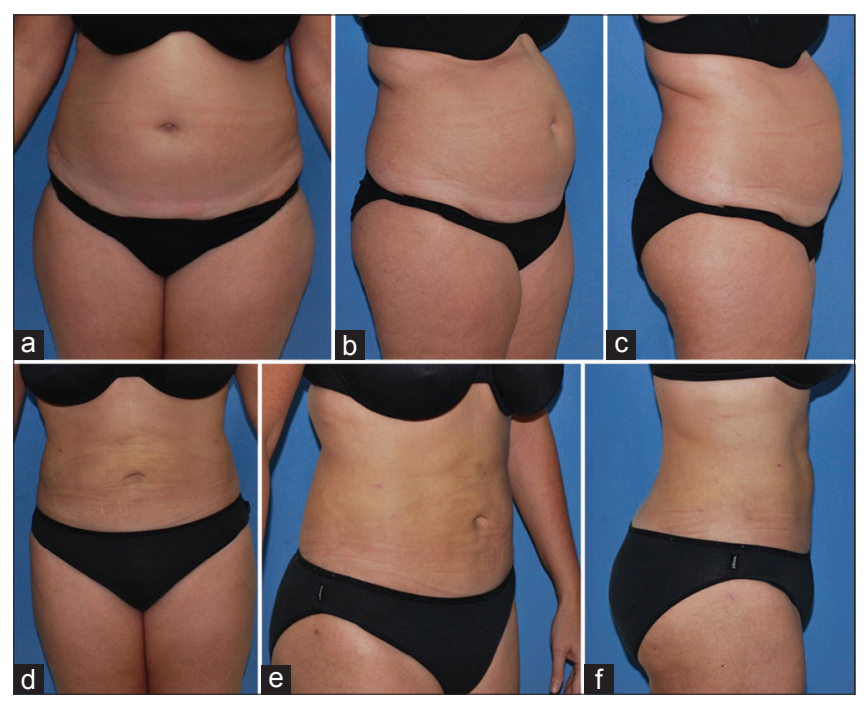

Figure 7: (a) Anterior preoperative views of 54 years old patient with body mass index 32. (b) Oblique Preoperative views of 54 years old patient with body mass index 32. (c) Lateral Preoperative views of 54 years old patient with body mass index 32. (d) Anterior Postoperative views after 6 months. (e) Oblique postoperative views after 6 months. (f) Lateral Postoperative views after 6 months

includes eight subunits, which we will discuss under the headings of anatomy, procedure and precautions.

\section{Zone 1 upper midline-overlying the linea alba from the xiphisternum to the umbilicus \\ Anatomy}

This unit is a narrow unit overlying the linea alba, in between the recti. Anatomically, there are tight fibrous adhesions between the skin and underlying rectus sheath. The groove is desirable by both men and women. In an athletic individual, it is well defined, but as weight increases, the groove disappears. The linea alba may also widen with diastasis of the recti in pregnancy and lead to an abnormally complex contour.

\section{Procedure}

This area is targeted for heavy liposuction (superficial and deep) to create a groove postoperatively.

\section{Precautions}

It is necessary to check for hernia sacs and divarication of recti. It is also important to use caution while creating the groove to avoid inadvertent perforation, as well as skin injury.

\section{Zone 2 upper rectus-overlying the rectus muscles from lower costal margin to the umbilicus}

\section{Anatomy}

The skin of this zone is more rigid and expands less than the lower abdomen. This is evidenced by the fact that there usually are not many stretch marks in this zone..$^{[9]}$ The fat is also compact and well arranged. There are natural adhesions in men in the form of horizontal transcriptions and in some athletic women as well. The skin is firmly adhered to the costal margins near the origin of the rectus muscles. As weight increases, there are the formation of folds and unnatural creases. Sometimes, even in thin individual creases are formed in the upper abdomen, which can be referred as 'flexion creases'. Excessive fat accumulation may cause pannus formation in the upper abdomen more often in women than men.

This region overlies the upper part of the rectus muscle. The recti have three tendinous intersections which are responsible for the appearance of six packs-at or just above the umbilicus, at the xiphoid, and midway between these two. These intersections may be in line or asymmetrical.

\section{Procedure}

Liposuction here is tailored in men to create six packs in men if needed. In women, the aim is to attain a concave shape. The lateral edge of this unit is targeted to create a linea semilunaris.

\section{Precautions}

This area is prone to skin injury, both blunt as well as excessive energy usage, particularly during groove creation. This area is also prone to contour irregularities in the hands of beginners. Furthermore, creating the appearance of muscles in an overweight man looks obviously fake and is referred to as the 'ninja turtle' man. There is also the risk of skin loss in this zone, particularly if there is an additional complication like seroma and overly tight fitting garment may aggravate it too.

\section{Zone 3 lower rectus-single unit over both the recti from the umbilicus to the upper pubic hairline \\ Anatomy}

The skin here is prone to laxity and panniculus formation. The skin usually forms a fold which stops at the inguinal region due to adhesions. The skin is poor quality with extensive stretch marks and poor capacity for retracting. ${ }^{[10]}$ In this region, the fat is divided by the scarpas fascia. That present below it is loosely arranged with sparse fibrous tissue. That present above it is compact with fibrous septae. The absence of posterior rectus sheath naturally gives a slightly convex shape in the lower abdomen. Rectus muscle converges in the lower part to insert on the pubic 
bones. The recti in this region are covered by the rectus sheath only on its anterior surface. There may be a partial tendinous intersection in the region of the arcuate line.

\section{Procedure}

During liposuction, it is preferable to maintain a slight bulge in this region.

\section{Precautions}

This area is also prone for skin injury. Moreover, special attention should be paid to postoperative compression in this region, due to its propensity for skin laxity. ${ }^{[11]}$ Seromas are also common here..$^{[12]}$

\section{Zone 4 lateral abdominal-overly the obliques, on either side of the recti from the costal margin to the inguinal ligament, till the mid-lateral line Anatomy}

The skin is usually thicker in this region unless there is a very large volume of fat accumulation. The skin is adherent in the region of the costal margin (Zone 4a) and the iliac crest (4b). Adhesions in men in the iliac crest region are to be preserved to avoid feminisation. In women, the mid-buttock area is responsible for a guitar deformity and needs special attention. Flexion creases often form with fat accumulation forming rolls or love handles that extend posteriorly.

Zone 4 overlies the oblique muscles of the abdomen and is bounded medially by Linea semilunaris. Linea semilunaris is a tendinous intersection formed by the aponeurosis of oblique muscles. The rectus muscle is thicker and has better support than the oblique muscles that causes a sort of 'ledge' at the Linea semilunaris. Or in other words, the musculofascial layer in this part is thinner than the central abdomen. There is a differential layer of fat deposition in this unit with an excessive layer of deep fatty tissue in the lower part (4b) and tougher fibrofatty tissue in the upper part (4a) near the coastal region.

\section{Procedure}

During liposuction, a concavity is created lateral to the recti to give an aesthetically pleasing effect as well as highlight the recti. It is also important to break down the fibrous tissue in the waistline as well as in any flexion creases. Higher energy would be needed in the fibrous upper part (4a) of this region while the lower part is easier (4b).

\section{Precautions}

Due to the relative weakness of the musculature in this region, it is prone to intraabdominal perforation, particularly while moving from a convex to a concave area. ${ }^{[13,14]}$ Similarly, one must be careful of intrathoracic perforation in the subcostal region. There have been reports of major vascular injury while operating in this region, by penetrating the iliac or femoral vessels. Failure to break down flexion creases will lead to recurrence of rolls with weight gain.

\section{Zone 5 pubic region-below the pubic hairline}

\section{Anatomy}

It is also called as 'mons pubis' or pubic mound overlying the pubic bones. The area consists of two layers of fat divided by the Scarpa's fascia. The youthful mons is narrow with good skin tone. With age, the hair-bearing skin of pubic region has tendency to sag with excess fat accumulation, and it covers the genitals in both men and women.

\section{Procedure}

Excess fat in this region should not be neglected during liposuction.

\section{Precautions}

This area is often neglected during liposuction, with the result that any excessive fat here becomes more pronounced following the surgery.

\section{Zone 6 lumbar-overly the lumbar muscles from the costal margin to the gluteal region, till the paraspinous muscles posteriorly \\ Anatomy}

The skin is quite thick in this region and very rarely has stretch marks. It has a good chance of contraction after liposuction. Fat deposition in this region obliterates the lumbar triangle and is generally in an oblique fashion. It has two compartments of fat deposits, superficial and deep. The deeper lies in the femoral triangle region. The lumbar region consists of the superior and an inferior lumbar triangle. The inferior triangle is more superficial and is a weak area with excessive fat accumulation. It is bounded by the latissimus Dorsi muscle, external oblique and iliac crest. Excess fat accumulation causes a distinct bulge referred to as 'love handles' or flanks. It also obliterates the dimple overlying the posterior superior iliac spine.

Hernias are known to occur in this region warranting extra precaution during liposuction procedure. Often, in massive weight loss patients, a bulge is persistent in this region due to musculofascial laxity. 


\section{Procedure}

Particular importance is to be paid to the love handles, as well as breaking down zones of fibrosis on either side to redrape the skin and create the waistline.

\section{Precautions}

The lumbar triangles are areas of weakness in an otherwise strong region. Attention should be paid to avoid inadvertent perforation. Aggressive superficial liposuction can permanently damage the dermal vasculature causing a mottled appearance..$^{[15]}$ Unequal liposuction of the love handles can cause postoperative asymmetry.

\section{Zone 7 posterior midline unit-overlies the spinous processes and the paraspinal muscles Anatomy}

Skin is adherent over the spinous processes and posterior iliac spine forming a midline groove and dimples. There is often a typical fat deposit inferiorly called the sacral pad which obliterates the curve posteriorly. It is also called as 'triangle of Venus'.

\section{Procedure}

Liposuction is performed mostly over the presacral region to improve the contour of the buttock in women. Some patents may require liposuction of midline if the groove is absent. It is important to leave some fat in the paraspinous region to aesthetically highlight the muscles.

\section{Precautions}

As it is a tough fibrous area, more force is needed which can cause injury to the skin or spinous processes.

\section{Zone 8 Bra roll-above the lumbar unit, extending into the infraaxillary region}

This region is of fat deposition, which extends from the lower margin of the breast posteriorly. The skin here is thick, and the fat is tough. It overhangs the subcostal region forming a flexion crease. Some patients may have multiple folds.

\section{Procedure}

This region is often overlooked, with the result that it stands out postoperatively, or it obliterates the waistline. Creases in between or below rolls need to be broken down to present recurrence.

\section{Precautions}

While operating this region in supine position, there is a risk of injury to latissimus Dorsi and its vascular pedicle as it forms the posterior axillary fold that falls flat on the table and the cannula may inadvertently enter the anterior surface of this muscle.

We used this system in 2000 cases and were able to achieve uniformly good results. Our success with this classification validates its use. The system was used in the following manner:

\section{Preoperative}

- To assess fat collection in different areas

- To plan liposuction and contouring in different areas-including etching.

\section{Intraoperative}

- To calculate the tumescent fluid requirement

- To calculate aspirate by region

- To compare both sides and avoid asymmetry

- To calculate ultrasonic energy usage bilaterally.

\section{Postoperative}

- To evaluate and report results

- To identify complications or fat residues by zone

- To track recurrence of fat with future weight gain

- We found the system of liposuction to be uniformly applicable and greatly improved our performance.

\section{CONCLUSION}

We report our system of aesthetic subunits of the trunk to share our experience and benefits. We have found this system to be easy to use, reliable and aid in evaluation, performance and reporting of liposuction results as well as avoiding complications.

\section{Financial support and sponsorship}

Nil.

\section{Conflicts of interest}

There are no conflicts of interest.

\section{REFERENCES}

1. Flynn TC, Coleman WP $2^{\text {nd }}$, Field LM, Klein JA, Hanke CW. History of liposuction. Dermatol Surg 2000;26:515-20.

2. Hoyos AE, Millard JA. VASER-assisted high-definition liposculpture. Aesthet Surg J 2007;27:594-604.

3. Matarasso A, Wallach SG. Abdominal contour surgery: Treating all aesthetic units, including the mons pubis. Aesthet Surg $J$ 2001;21:111-9.

Indian Journal of Plastic Surgery Volume 51 Issue 2 May-August 2018 
4. González-Ulloa M. Regional aesthetic units of the face. Plast Reconstr Surg 1987;79:489-90.

5. Spear SL, Davison SP. Aesthetic subunits of the breast. Plast Reconstr Surg 2003;112:440-7.

6. Mendieta CG. Gluteoplasty. Aesthet Surg J 2003;23:441-55.

7. Mentz HA $3^{\text {rd }}$, Gilliland MD, Patronella CK. Abdominal etching: Differential liposuction to detail abdominal musculature. Aesthetic Plast Surg 1993;17:287-90.

8. Ersek RA, Salisbury AV. Abdominal etching. Aesthetic Plast Surg 1997;21:328-31.

9. Cua AB, Wilhelm KP, Maibach HI. Elastic properties of human skin: Relation to age, sex, and anatomical region. Arch Dermatol Res 1990;282:283-8.

10. Dabb RW, Hall WW, Baroody M, Saba AA. Circumferential suction lipectomy of the trunk with anterior rectus fascia plication through a periumbilical incision: An alternative to conventional abdominoplasty. Plast Reconstr Surg 2004;113:727-32.

11. Nagy MW, Vanek PF Jr. A multicenter, prospective, randomized, single-blind, controlled clinical trial comparing VASER-assisted lipoplasty and suction-assisted lipoplasty. Plast Reconstr Surg 2012;129:681e-9e.

12. Katz BE, Bruck MC, Felsenfeld L, Frew KE. Power liposuction: A report on complications. Dermatol Surg 2003;29:925-7.

13. Mallappa M, Rangaswamy M, Badiuddin MF. Small intestinal perforation and peritonitis after liposuction. Aesthetic Plast Surg 2007;31:589-92.

14. Di Candia M, Malata CM. Aesthetic and functional abdominal wall reconstruction after multiple bowel perforations secondary to liposuction. Aesthetic Plast Surg 2011;35:274-7.

15. Porto da Rocha R, Porto da Rocha EL, de Souza Pinto EB, Sementilli A, Nakanishi CP. Cutis marmorata resemblance after liposuction. Aesthetic Plast Surg 2005;29:310-2. 